\title{
Nobiletin inhibits epithelial-mesenchymal transition of human non-small cell lung cancer cells by antagonizing the TGF- $\beta 1 /$ Smad3 signaling pathway
}

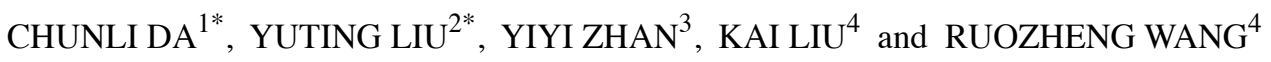 \\ Departments of ${ }^{1}$ Intensive Care,${ }^{2}$ Anesthesiology, ${ }^{3}$ Chemotherapy for Lung Cancer, ${ }^{4}$ Radiotherapy for the Head and Neck, \\ Tumor Hospital of Xinjiang Medical University, Urumqi, Xinjiang 830000, P.R. China
}

Received November 20, 2015; Accepted January 7, 2016

DOI: $10.3892 /$ or.2016.4661

\begin{abstract}
Epithelial-mesenchymal transition (EMT) is a critical cellular process in cancer metastasis, during which epithelial polarized cells become motile mesenchymal cells. Since transforming growth factor- $\beta$ (TGF- $\beta$ ) is a potent inducer of EMT, blocking of TGF- $\beta /$ Smad signaling has become a promising cancer therapy. Nobiletin, a polymethoxy flavonoid from Citrus depressa, has been shown to be valuable for cancer treatment, yet the mechanism remains unclear. In the present study, lung adenocarcinoma A549 and H1299 cells were used to evaluate the effect of nobiletin on EMT induced by TGF- $\beta 1$. Nobiletin successfully inhibited TGF- $\beta 1$-induced EMT, migration, invasion and adhesion in vitro, accompanied by attenuation of MMP-2, MMP-9, p-Src, p-FAK, p-paxillin, Snail, Slug, Twist and ZEB1 expression. Nobiletin inhibited the transcriptional activity of Smads without changing the phosphorylation status or translocation of Smads induced by TGF- $\beta 1$. Moreover, Smad 3 is requisite in TGF- $\beta 1$-stimulated EMT. Smad3 overexpression meaningfully impaired the ability of nobiletin to reverse TGF- $\beta 1$-induced EMT. In vivo, nobiletin prohibited the growth of metastatic nodules in the lungs of nude mice. Moreover, nobiletin inhibited tumor growth and reversed EMT in mice bearing A549-Luc xenografts, as revealed by IVIS imaging and immunohistochemical analysis. Collectively, the data suggest that nobiletin prevents EMT by inactivating TGF- $\beta 1 / \mathrm{Smad} 3$ signaling.
\end{abstract}

Correspondence to: Dr Ruozheng Wang, Department of Radiotherapy for the Head and Neck, Tumor Hospital of Xinjiang Medical University, Xinshi, 789 Suzhou Road, Urumqi, Xinjiang 830000, P.R. China

E-mail:wrz8526@163.com

*Contributed equally

Key words: nobiletin, epithelial-mesenchymal transition, invasion, TGF- $\beta /$ Smads, NSCLC

\section{Introduction}

Non-small cell lung carcinoma (NSCLC) is a leading cause of cancer-related death worldwide, comprising $75-80 \%$ of all lung cancer cases. Owing to early metastasis and the lack of efficient therapeutic treatment, the overall 5-year survival rate for lung cancer patients is less than $15 \%$ (1). Growing evidence demonstrates that epithelial-mesenchymal transition (EMT) plays a crucial role in tumorigenesis, cancer recurrence and metastasis $(2,3)$. Correct activation of the EMT program is essential for physical development; however, irregular EMT induction may disturb epithelial homeostasis and tissue integrity, leading to unexpected disease pathologies (4). Specifically, EMT endows tumor cells with more invasive properties. As a driver of tumor invasion, EMT stimulates cells to spread from a primary organ site, migrate through blood and lymphatic vessels, extravasate into the surrounding tissue, and ultimately to propagate into tumors at distant organs (5). Moreover, research has proven that EMT is closely related with poor prognosis in NSCLC. Hence, illustrating the mechanisms underlying EMT and developing clinical approaches to target EMT for NSCLC patients is of great value, although identifying definite therapeutic targets and optimal patient populations remains a great challenge (6).

The process of EMT consists of three major steps in cancer: i) loss of cell-cell junctions and a decrease in the epithelial marker E-cadherin; ii) acquisition of the mesenchymal marker $\mathrm{N}$-cadherin; and iii) cytoskeleton rearrangement intended for invasive properties (7). Additionally, these changes are paralleled with secretion of matrix metalloproteinase-2/-9 (MMP-2/-9) and focal adhesion kinase (FAK). MMP-2/-9, proteolytic enzymes that degrade and modify the extracellular matrix (ECM), act directly on cell surface molecules and activate EMT (8). FAK is a non-receptor tyrosine kinase primarily which localizes to cell-matrix adhesions and acts as a central regulator of focal adhesion to influence cell adhesion and metastasis. p-FAK has been observed in invasive tumors, and is closely related with E-cadherin imbalance during EMT (9).

EMT occurs under several pathological conditions and is regulated by multiple pathways. Among which, TGF- $\beta$ is regarded as the most characterized factor responsible for 
EMT initiation. As a multifunctional cytokine, TGF- $\beta$ takes part in many biological processes including cell proliferation, adhesion and migration. By far, three members of the TGF- $\beta$ family have been identified; TGF- $\beta 1,2$ and 3, and EMT has been demonstrated to be highly correlated with TGF- $\beta 1$ (10). TGF- $\beta 1$ facilitates the phosphorylation of Smad2 and Smad3, and phospho-Smad2 and phospho-Smad3 form heteromeric complexes with Smad4. The translocation of Smad2/3/4 complexes into the nucleus eventually affects target gene expression (11). Consequently, disturbance of $\mathrm{Smad} 2 / 3$ activation may block TGF/Smad signaling and subsequent EMT.

Nobiletin (5,6,7,8,3',4'-hexamethoxyflavone) (Fig. 1A) is a major component in citrus fruits, particularly in the peels of sweet oranges (Citrus sinensis) (12). Nobiletin was found to exhibit antimetastatic activities as indicated by regulating pro-MMP-1/-9 and TIMP-1 expression levels in human fibrosarcoma cells, and the suppression of invasion and migration in human gastric adenocarcinoma cells via reducing the expression of MMP-2/-9 and FAK (13). Moreover, nobiletin has the potential to inhibit the metastasis of breast cancer via reducing expression levels of CXC chemokine receptor type 4 (CXCR4) and MMP-9 (14). Gao et al (15) reported that nobiletin has a distinct ability to suppress hypoxia-induced EMT in lung cancer cells. In the present study, we provide important evidence that TGF- $\beta 1 / \mathrm{Smad} 3$ signaling is a vital mechanism that can drive EMT and metastasis in lung adenocarcinoma. Nobiletin can inhibit TGF- $\beta 1$-induced EMT by blocking Smad3 transcription, signifying a potential use of nobiletin in the treatment of lung adenocarcinoma.

\section{Materials and methods}

Cell culture and viability assay. Human adenocarcinoma A549 and H1299 cells (Sigma-Aldrich, St. Louis, MO, USA) were grown in Dulbecco's modified Eagle's medium (DMEM) supplemented with $10 \%$ fetal bovine serum (FBS), $100 \mathrm{IU} / \mathrm{ml}$ penicillin and $100 \mu \mathrm{g} / \mathrm{ml}$ streptomycin. Cellular viability was measured by MTT reduction assay. MTT solution $(5 \mathrm{mg} / \mathrm{ml})$ was added to each well. After a 4-h incubation, MTT solution was removed and isopropanol-hydrochloric acid (25:1) was added to dissolve the formed formazan crystals. The absorbance was measured at $570 \mathrm{~nm}$ against a $690 \mathrm{~nm}$ reference with a microplate reader.

Adhesion and Transwell migration assays. For adhesion assays, A549 and H1299 cells were treated with TGF- $\beta 1$ $(5 \mathrm{ng} / \mathrm{ml})$ or/and nobiletin for $24 \mathrm{~h}$. Cells were suspended in DMEM and plated at a density of $1 \times 10^{4}$ cells/well onto 96-well plates coated with collagen IV. Cells were then fixed with methanol and stained with $0.1 \%$ crystal violet and absorbance was read at $595 \mathrm{~nm}$ spectrophotometrically.

For the scratch assays, A549 cells were plated in 24-well tissue culture plates and allowed to reach confluency. At this point a scratch was introduced into the monolayer using a sterile 200- $\mu 1$ pipette tip. The cells were then washed in warm phosphate-buffered saline (PBS) to remove the debris and the medium was replaced with either fresh medium (control samples) or medium containing TGF- $\beta 1(5 \mathrm{ng} / \mathrm{ml})$ or/and nobiletin $(10$ and $20 \mu \mathrm{M})$ for $24 \mathrm{~h}$. Three independent experiments were carried out for each experimental condition.
The migration assay was performed using 24-well 8- $\mu \mathrm{m}$ pore Transwell inserts (Corning), with the upper face of polycarbonate filters coated with Matrigel. Cells $\left(1 \times 10^{5}\right)$ were added to the upper chamber. The lower chambers were filled with $20 \%$ FBS/DMEM media to serve as a chemoattractant. The non-migrated cells in the upper chamber were removed gently by a cotton swab at $24 \mathrm{~h}$ following reseeding, whereas, the migrated cells in the lower side were stained with $0.1 \%$ crystal violet. The mean of six fields from three separate trials was used to calculate the average number of migratory cells.

Immunofluorescence assay. Immunofluorescence assay was performed as previously described (16). In brief, cultured cells grown on plastic chamber slides were fixed in $4 \%$ formaldehyde for $30 \mathrm{~min}$ at room temperature. Then the cells were permeabilized in $0.2 \%$ Triton X-100 and blocked with $10 \%$ goat serum, and then incubated overnight at $4^{\circ} \mathrm{C}$ with the primary antibodies (diluted 1:100 in blocking buffer) against $\mathrm{E}$-cadherin and $\mathrm{N}$-cadherin. Afterwards, the sample was washed with PBS containing blocking buffer and incubated with the fluorophore-conjugated secondary antibody (Abcam). Cover glasses were counterstained with 4',6-diamidine-2'-phenylindole dihydrochloride (DAPI) $(5 \mathrm{mg} / \mathrm{ml}$; Invitrogen Life Technologies). Fluorescence images were captured using a fluorescence microscope (Carl Zeiss, Göttingen, Germany).

Transient transfection. PcDNA3.1-Flag-Smad3 plasmid was prepared using Qiagen plasmid DNA preparation kit. Plasmid or Smad2/3 small interfering RNA (siRNA) (Santa Cruz Biotechnology, Santa Cruz, CA, USA) transient transfection of A549 cells was performed according to the protocol of the Lipofectamine $2000^{\mathrm{TM}}$ reagent (Invitrogen, Carlsbad, CA, USA).

Western blot analysis. A549 and $\mathrm{H} 1299$ cells were lysed at $4{ }^{\circ} \mathrm{C}$ (30 min) in Tris-buffered saline. Whole lysates were analyzed by SDS-PAGE, and transferred to polyvinylidene difluoride (PVDF) membranes. The membranes were blocked with 5\% skim milk with Tween-20 and probed with primary antibodies against E-cadherin, N-cadherin, vimentin, Snail, Slug, ZEB1, Twist, Src, FAK and $\beta$-actin (Thermo Fisher Scientific). Bound antibodies were conjugated with horseradish peroxidase secondary antibodies, and the membranes were developed with chemoluminescence reagents. Protein expression was semi-quantified by densitometric analysis.

RNA extraction and RT-PCR. Total RNA was isolated from A549 cells using the total RNA extraction kit as described by the manufacturer (Life Technologies, Inc., Grand Island, NY, USA). RT-PCR was performed using the Qiagen RT-PCR kit. The appropriate primers (MMP-2, 5'-GGCCCTGTCACTC CTGAGAT-3' and 5'-GGCATCCAGGTTATCGGGGA-3'; MMP-9, 5'-AGGCCTCTACAGAGTCTTTG-3' and 5'-CAG TCCAACAAGAAAGGACG-3'; GADPH, 5'-GGCCTCCA AGGAGTAAGACC-3' and 5'-AGGGGTCTACATGGCA ACTG-3') were used for PCR amplifications under the following conditions: 30 cycles at $95^{\circ} \mathrm{C}$ for $1 \mathrm{~min}, 60^{\circ} \mathrm{C}$ (MMP-2 and GAPDH) or $59^{\circ} \mathrm{C}$ (MMP-9) for $1 \mathrm{~min}, 72^{\circ} \mathrm{C}$ for $1 \mathrm{~min}$ and $72^{\circ} \mathrm{C}$ for $10 \mathrm{~min}$. The PCR products were separated by $1.5 \%$ agarose gel and visualized by ethidium bromide staining. 


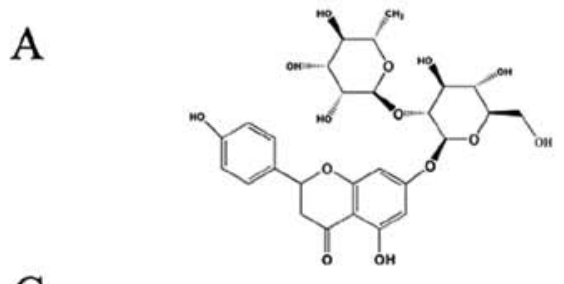

$\mathrm{C}$
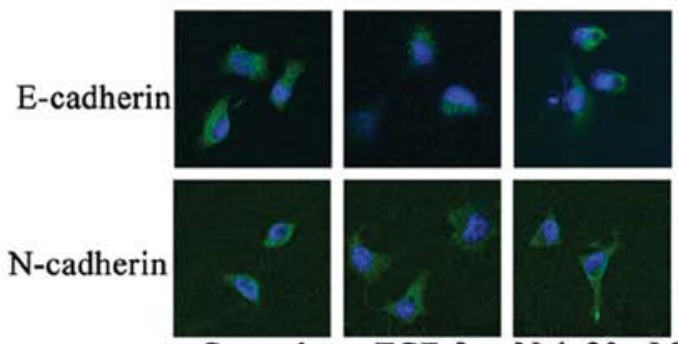

Control
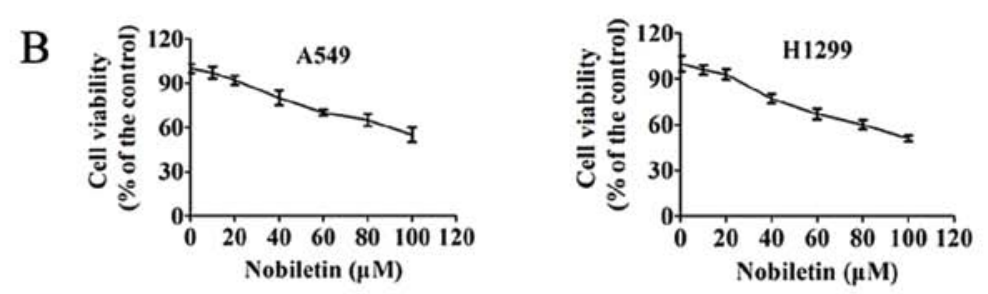

A549

D

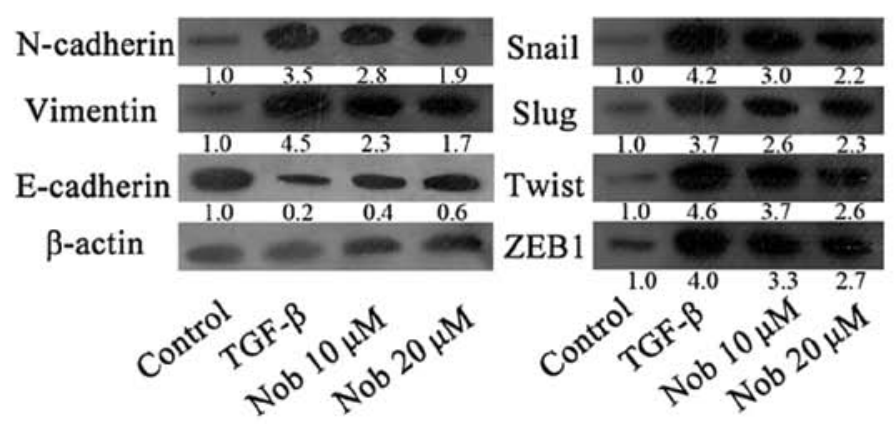

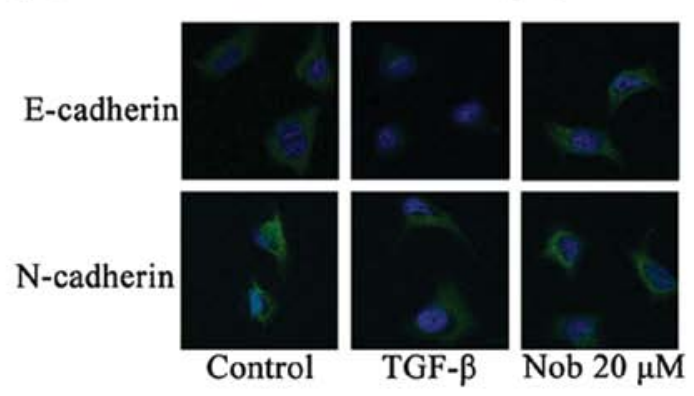

H1299

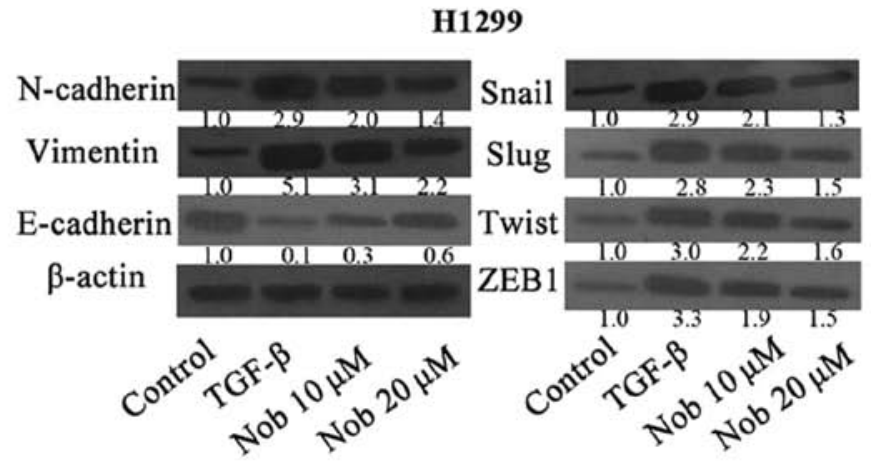

Figure 1. Nobiletin inhibits TGF- $\beta 1$-induced EMT in A549 and H1299 cells. (A) Chemical structure of nobiletin. (B) A549 and H1299 cells were treated with TGF- $\beta 1(5 \mathrm{ng} / \mathrm{ml})$ or/and nobiletin (Nob) for $24 \mathrm{~h}$, and then cell viability was determined with the MTT assay. Values are expressed as the mean \pm SD from triplicates. ${ }^{~} \mathrm{P}<0.05$, compared with the control. ${ }^{*} \mathrm{P}<0.05$, compared with the cells treated with TGF- $\beta 1$ only. (C) The expression levels of epithelial marker, E-cadherin, and mesenchymal marker, $\mathrm{N}$-cadherin, were evaluated by immunofluorescence. (D) EMT-related proteins were assessed by western blotting.

In vivo xenograft metastasis and tumor growth experiments. All mice were obtained from the SLAC Laboratory Animal Center of Shanghai. Ethical approval was obtained for the use of the animals, and all experiments were performed in accordance with the Guidelines for Animal Care of the Institutional Animal Care and Use Committee of the Tumor Hospital of Xinjiang Medical University. i) Five-week-old nude mice

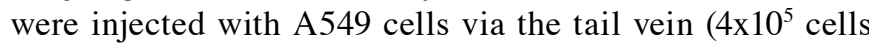
in $0.2 \mathrm{ml} \mathrm{PBS}$ ). Nobiletin (20 and $40 \mathrm{mg} / \mathrm{kg}$ ) was orally administered once daily. Cyclophosphamide $(20 \mathrm{mg} / \mathrm{kg})$ was used as the positive control, injected intraperitoneally every three days. Saline was administered orally every day for the control group ( $\mathrm{n}=8$ mice/group). After 42 days, the mice were sacrificed. After fixation in Bouin's solution, the number of lung metastases were confirmed under a dissecting microscope. The lungs were further subjected to hematoxylin and eosin (H\&E) staining. ii) C57BL/6 mice were injected with Lewis cells via the tail vein $\left(8 \times 10^{5}\right.$ cells in PBS) to establish a metastatic model. Administration was the same as the first model. After 15 days, the mice were sacrificed and lung metastatic nodules were confirmed under a microscope. iii) Nude mice were implanted subcutaneously with A549-Luc cells. Administration was the same as the first model. Thirty days after cell implantation, imaging was performed with an IVIS imaging system (Caliper Life Sciences, Inc., Hopkinton, MA,
USA), and nude mice were sacrificed and tumors were excised and weighed.

Immunohistochemistry. Immunohistochemistry of lung tissue was performed as previously reported (17). Formalin-fixed, paraffin-embedded tissue sections were cut at a thickness of $4 \mu \mathrm{m}$. Antigen recovery was performed by heating in $0.01 \mathrm{M}$ sodium citrate buffer at $80^{\circ} \mathrm{C}$ for $30 \mathrm{~min}$ using a microwave oven. Endogenous peroxidase activity and non-specific staining were blocked with the kit reagents. The tissues were incubated overnight with the primary E-cadherin and N-cadherin antibodies. Tissues were then incubated with peroxidase-bound IgG. The slides were counterstained with hematoxylin. Mounted tissues were imaged on an Olympus microscope. The numbers of E-cadherin- or N-cadherin-positive cells were determined at a magnification of $\mathrm{x} 400$ in three segments/slide.

Statistical analysis. Statistical analysis was performed using ANOVA, $\chi^{2}$ or Student's t-test using SPSS 16.0 software. Statistical significance was assigned at $\mathrm{P}<0.05$.

\section{Results}

Nobiletin rebalances EMT-related genes. An MTT assay was used to determine the non-cytotoxic concentrations of 
A

A549

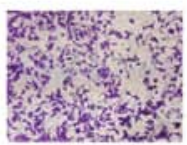

H1299

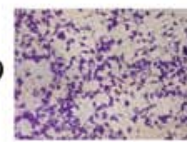

Control
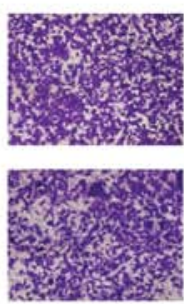

TGF- $\beta$
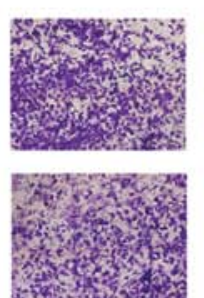

Nob $10 \mu \mathrm{M}$ Nob $20 \mu \mathrm{M}$
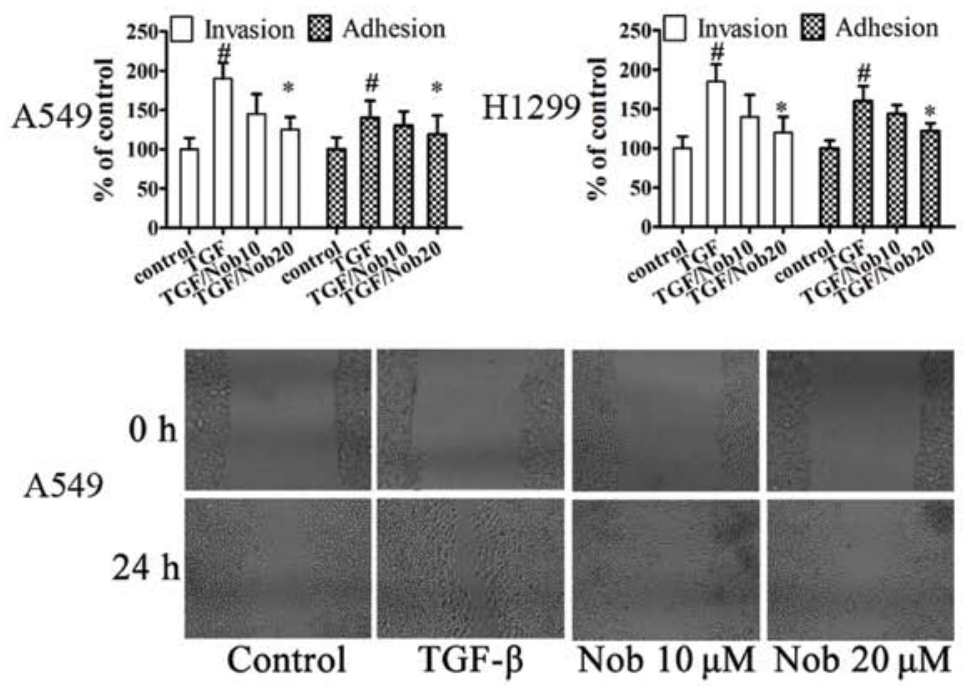

B
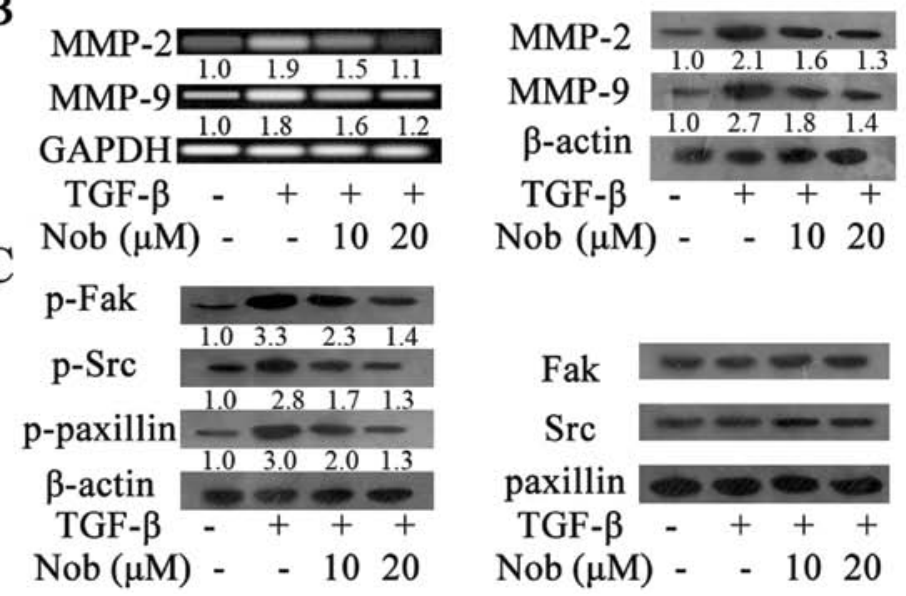

Figure 2. Nobiletin inhibits TGF- $\beta 1$-induced adhesion, migration and invasion in lung adenocarcinoma cells. (A) The effect of nobiletin (Nob) on TGF- $\beta 1$-induced migration, invasion and adhesion. The invasive ability of A549 and H1299 cells was quantified by counting the number of cells that invaded to the underside of the porous membrane. Images were captured under phase contrast microscope. ${ }^{*} \mathrm{P}<0.05$, compared with the control. ${ }^{*} \mathrm{P}<0.05$, compared with the cells treated with TGF- $\beta 1$ only. (B) Effects of nobiletin on TGF- $\beta 1$-induced expression of MMP- 2 and MMP-9 were measured by RT-PCR and western blotting, respectively. (C) p-FAK, p-Src, p-paxillin levels were assessed by western blotting.

nobiletin $(0-100 \mu \mathrm{M})$ in the presence of TGF- $\beta 1(5 \mathrm{ng} / \mathrm{ml})$ for $24 \mathrm{~h}$. According to the MTT assays (Fig. 1B), concentrations of 10 and $20 \mu \mathrm{M}$ were selected for further study, which exhibited no significant toxicity on cell viability. EMT is a crucial step in tumor metastasis, characterized by polarity and epithelial markers, as well as motile mesenchymal cells. Specifically, E-cadherin is a negative EMT biomarker, while $\mathrm{N}$-cadherin is a mesenchymal biomarker. As shown in the immunofluorescence assay, TGF- $\beta 1$-stimulated A549 and H1299 cells exhibited characteristic N-cadherin increase and E-cadherin decrease (Fig. 1C). However, nobiletin rebalances EMT-related genes disturbed by TGF- $\beta 1$. In addition, similar results were obtained from the protein assays (Fig. 1D). The most prominent E-cadherin regulators are Snail-related zinc-finger transcription factors (Snail and Slug), ZEB1 and Twist. TGF- $\beta 1$ treatment increased the expression of Snail, Slug, ZEB1 and Twist, and the increase was reversed by nobiletin treatment (Fig. 1D).

Nobiletin prohibits the metastatic capacity of lung adenocarcinoma cells along with the downregulation of MMP-2 and MMP-9 and the FAK/Src pathway. Nobiletin suppressed both A549 and H1299 cell invasion in the transmembrane migration assay induced by TGF- $\beta 1$ (Fig. 2A). Moreover, nobiletin 
A

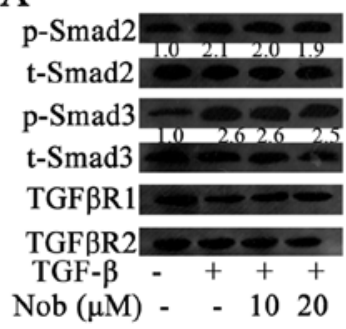

B

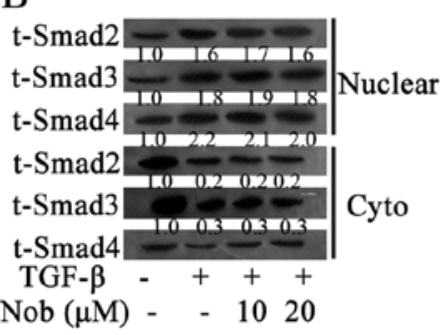

$\mathrm{C}$

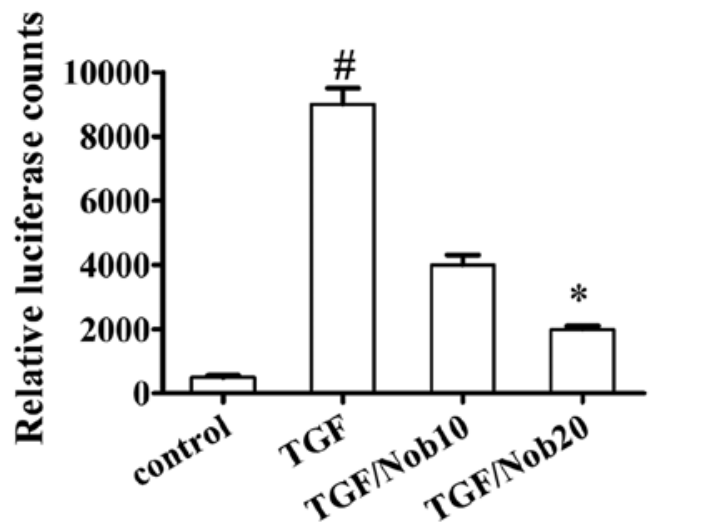

Figure 3. Nobiletin abrogates Smad transcriptional activity induced by TGF$\beta 1$. A549 cells were incubated with TGF- $\beta(5 \mathrm{ng} / \mathrm{ml})$ with or without nobiletin (Nob) for $24 \mathrm{~h}$. (A) The expression levels of TGF $3 \mathrm{R} 1$, TGF $\beta$ R2, p-Smads and total-Smads were assessed by western blotting. (B) Cytoplasmic (Cyto) and nuclear fractions were prepared to examine the translocation of Smads by western blotting. (C) After A549-SBE-Luc cells were treated with TGF- $\beta$ or/ and nobiletin, luciferase expression was measured and normalized to the protein concentrations. ${ }^{*} \mathrm{P}<0.05$, compared with the control; " $\mathrm{P}<0.05$, compared with the cells treated with TGF- $\beta 1$ only.

inhibited the capacity of A549 and H1299 cells to adhere to wells coated with human collagen type IV. After TGF- $\beta 1$ stimulation, A549 cells displayed enhanced wound closure activity and almost filled the scratched area, whereas the wound still displayed an obvious gap in the nobiletin-pretreated group.

RT-PCR and western blot assays were applied to examine the mRNA and protein levels of MMP-2/-9. An increase in the mRNA and protein levels of MMP-2/-9 was observed following TGF- $\beta 1$ incubation, while nobiletin treatment abrogated the increase (Fig. 2B). Activation of FAK is normally induced by various growth factors, including TGF- $\beta$. Therefore, we further explored the effect of nobiletin on the activation of focal adhesion-related signaling molecules, FAK, Src and paxillin. As shown in Fig. 2C, TGF- $\beta 1$ induced the phosphorylation of FAK, Src and paxillin and these phosphorylation events were inhibited by nobiletin.

Nobiletin prevents the transcriptional activity of Smads induced by TGF- $\beta$. In the TGF- $\beta 1 /$ Smad signaling pathway, TGF- $\beta R 2$ induces recruitment and phosphorylation of TGF- $\beta R 1$ after binding to TGF- $\beta 1$ (18). Both TGF $\beta$ R 1 and TGF $\beta$ R2 can be detected in A549 cells. As expected, the expression of TGF $\beta R 1$ and TGF $\beta R 1$ did not change following either TGF- $\beta 1$ or nobiletin treatment. TGF- $\beta 1$-triggered EMT is Smad-dependent. The activated TGF- $\beta$ R 1 subsequently induces phosphorylation of $\operatorname{Smad} 2 / 3$. Therefore, western blot analysis was carried out to examine whether nobiletin influences Smad phosphorylation. TGF- $\beta 1$-treated cells
A
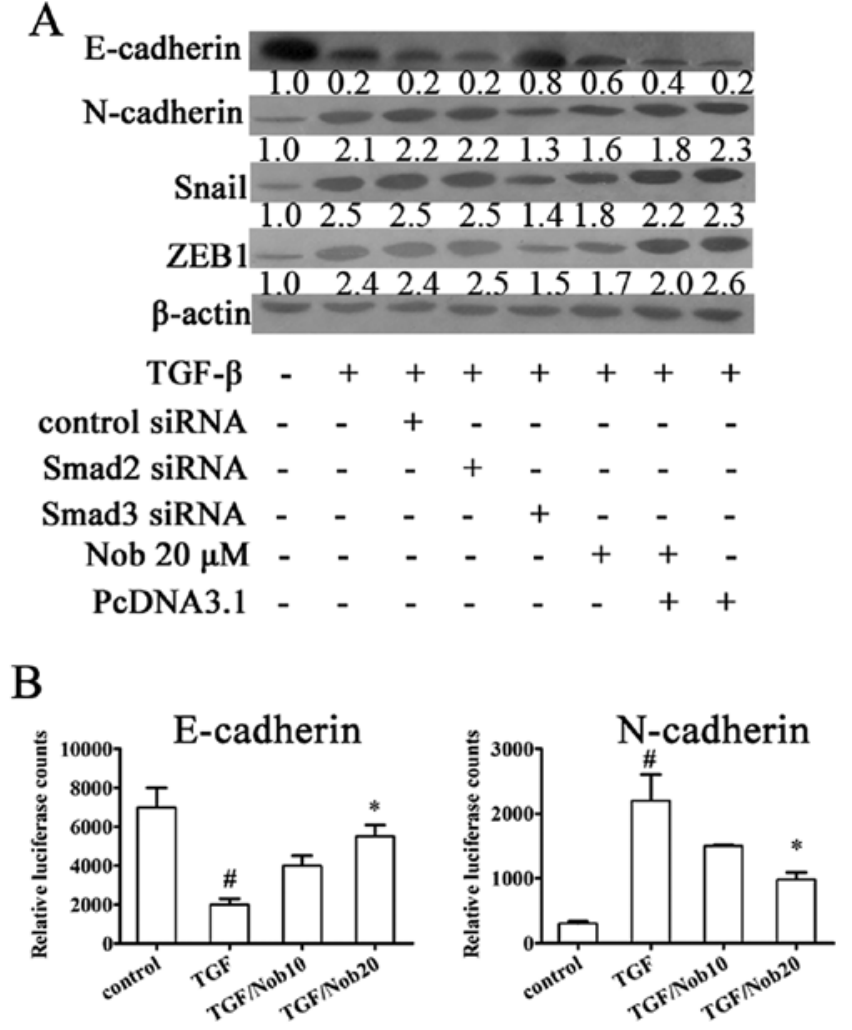

Figure 4. Nobiletin suppresses EMT via the TGF- $\beta 1 / \mathrm{Smad} 3$ signaling pathway. (A) Smad2 siRNA, Smad3 siRNA or PcDNA3.1-Flag-Smad3 was transfected into the A549 cells, respectively. After the A549 cells were treated with TGF- $\beta 1$ and nobiletin (Nob) for 24 h, E-cadherin, N-cadherin, Snail and ZEB1 were detected by western blotting. (B) The promoter expression of E-cadherin and $\mathrm{N}$-cadherin after nobiletin treatment. " $\mathrm{P}<0.05$, compared with the control; ${ }^{*} \mathrm{P}<0.05$, compared with the cells treated with TGF- $\beta 1$ only.

showed aggravated phosphorylation of $\mathrm{Smad} 2 / 3$ after a $60-\mathrm{min}$ incubation, and the increase in phosphorylation was not affected after nobiletin exposure (Fig. 3A). Phosphorylated Smad2 and Smad3 form heteromeric complexes with the mediator Smad4. These Smad2/3/4 complexes translocate to the nucleus, and bind to the Smad binding elements (SBEs). Nobiletin failed to prevent Smads from translocating into the nucleus, as induced by TGF- $\beta$ (Fig. 3B). Transcriptional activity of Smad was also examined. A SBE-luciferase reporter plasmid was stably transfected into A549 cells to evaluate the luciferase activity. Consistent with previous studies, TGF- $\beta 1$ considerably augmented the luciferase-reporter activity of Smad, which was greatly abolished by nobiletin treatment (Fig. 3C). These data infer that nobiletin prohibited EMT by eliminating the transcriptional activity of Smads, rather than influencing the translocation or phosphorylation.

Nobiletin suppresses EMT via the TGF- $\beta 1 /$ Smad3 signaling pathway. In order to explore the detailed mechanism of nobiletin in regards to EMT, we transfected Smad2 siRNA and Smad3 siRNA into A549, and then detected the EMT-related protein levels. The protein expression of E-cadherin was significantly upregulated while N-cadherin, Snail and ZEB1 were downregulated due to Smad3 knockdown. In contrast, Smad2 siRNA had little or negligible effect on the expression of EMT-related proteins. In the nobiletin-treated group, these 
A

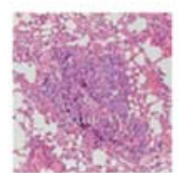

Control

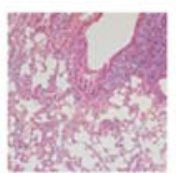

Cy 20

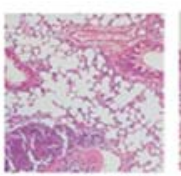

Nob 20
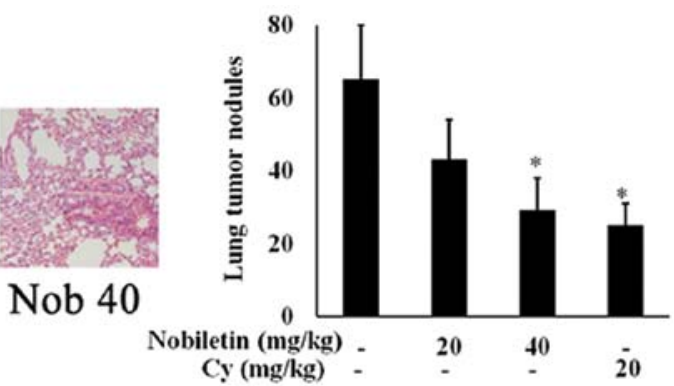

B

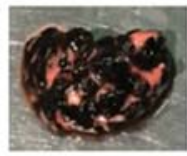

Control

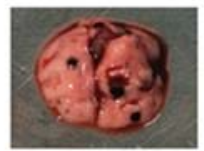

Cy 20

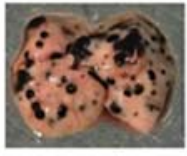

Nob 20

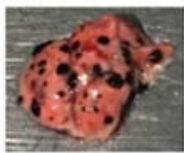

Nob 40

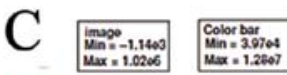

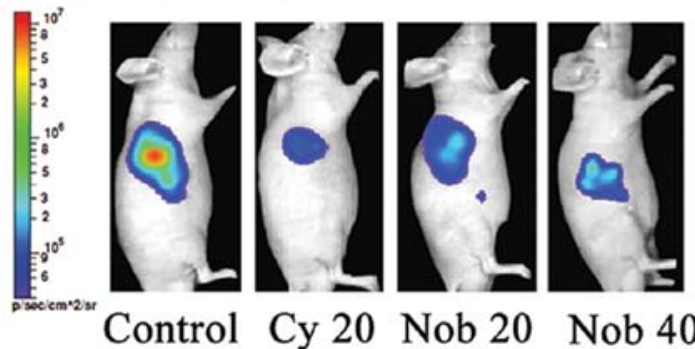

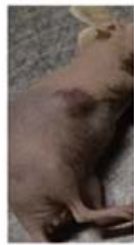
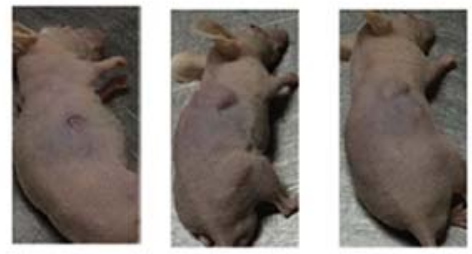

Control

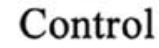

Cy 20
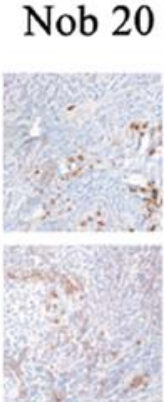

Nob 40

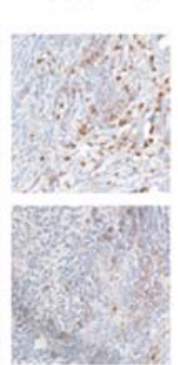

Control

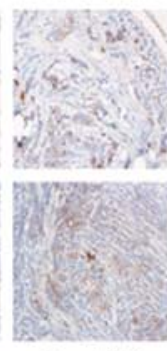

Cy 20
Nob 20

Nob 40

Figure 5. Nobiletin decrease metastasis and tumor growth in vivo. (A) The effect of nobiletin on lung metastasis in nude mice injected with A549 cells via tail vein. Tumor metastasis in lung tissue was present as indicated by the H\&E staining assay. The number of lung metastatic nodules on the surface was quantified. (B) The effect of nobiletin on lungs metastasis in C57BL/6 mice injected with Lewis cells via tail vein. Representative lung tissue images are shown. (C) The effect of nobiletin on tumor growth and EMT markers in nude mice implanted subcutaneously with A549-Luc cells. At the end of 30 days of treatment, the tumor volumes of nude mice bearing A549 xenografts were measured. Luciferase was assessed using bioluminescence instrument. Representative images are shown. Tumor samples were then subjected to immunohistochemical analysis to examine the expression of $\mathrm{E}$-cadherin and $\mathrm{N}$-cadherin. ${ }^{* *} \mathrm{P}<0.01$; ${ }^{*} \mathrm{P}<0.05$, compared with saline-treated controls. Nob, nobiletin; Cy, cyclophosphamide.

protein expression levels exhibited a similar tendency compared with the Smad3 siRNA-transfected A549 cells (Fig. 4A). To further confirm the interaction between nobiletin and Smad3, A549 cells were transfected with PcDNA3.1-Flag-Smad3. Accordingly, the expression of E-cadherin was decreased, while N-cadherin, Snail and ZEB1 were increased. Smad3 overexpression considerably reduced the ability of nobiletin to inhibit the TGF- $\beta 1$-induced increase in $\mathrm{N}$-cadherin and to reverse the TGF- $\beta$-stimulated decrease in E-cadherin. These data reveal the essential role of Smad3 in EMT progression and propose that Smad3 is involved in the inhibitory effects of nobiletin on EMT. We further examined the promoter expression of E-cadherin and N-cadherin. Nobiletin treatment resulted in the reversal of the E-cadherin promoter decrease as well as the suppression of N-cadherin promoter increase (Fig. 4B). Since the promoters of E-cadherin and $\mathrm{N}$-cadherin are dependent on Smad3, we propose that nobiletin obstructs EMT by blocking Smad3-dependent transcriptional activity.

Nobiletin decreases metastasis and tumor growth in vivo. To further explore the antimetastatic potential of nobiletin in vivo, two experimental metastatic models of lung cancer were applied. One model was established by injecting A549 cells in nude mice via the tail vein. Nobiletin (20 and $40 \mathrm{mg} / \mathrm{kg}$ ) was administered orally once daily. Cyclophosphamide was used as the positive control. As shown in Fig. 5A, the number of lung metastatic nodules was $65 \pm 15$ in the control group on day 42 , while there were only $43 \pm 11$ and $29 \pm 9$ nodules in the 
20 and $40 \mathrm{mg} / \mathrm{kg}$ group, respectively ( $\mathrm{P}<0.05$, vs. the control group). As depicted in the H\&E staining, tumor cells were organized as nests, with necrosis in the center of the metastatic area. Metastasis was less severe in the nobiletin-treated group, suggesting that nobiletin markedly inhibited metastasis in vivo. Similar results were observed in another experimental metastatic model, which was established by injecting Lewis cells into C57BL/6 mice via the tail vein. Representative lung tissue images are shown in Fig. 5B.

Tumor xenografts transplanted by A549-Luc cells were applied to assess the antitumor effect of nobiletin in vivo. IVIS imaging results revealed strong suppression of tumor volume in the nobiletin-treated nude mice $(\mathrm{P}<0.01$ vs. the control group) (Fig. 5C). In the IHC analysis, nobiletin facilitated E-cadherin protein expression, and inhibited $\mathrm{N}$-cadherin protein. All these results suggest that nobiletin suppressed EMT and the growth of lung cancer cells in vivo.

\section{Discussion}

Despite advancements in the diagnosis of NSCLC along with revolutionary progress in targeted therapies, treatment of NSCLC after distant organ metastasis remains a great challenge. Various plant or fruit-derived agents with few side-effects have been chosen as potential alternatives for the therapy of lung cancer. Citrus species is a natural product containing phytochemicals with promise in cancer therapy. Numerous studies have demonstrated the citrus potential as a chemopreventive agent. As well, nobiletin has anticancer activity in various malignant tumors such as gastric, lung, colon and nasopharyngeal cancers. TGF- $\beta$ is a multifunctional secreted polypeptide that signals through receptor serine/threonine kinases. Accumulating evidence has revealed that TGF- $\beta$ stimulates metastasis and invasion in various tumors. TGF- $\beta$ has received much attention as a characterized inducer of EMT during cancer progression and metastasis (19). Upon activation of the TGF- $\beta$ pathway, cancer cells undergo evident and irreversible EMT, finally resulting in more aggressive and metastatic tumors. In the present study, we demonstrated that nobiletin suppresses adhesion, EMT, metastasis and invasion of lung cancer via antagonizing the TGF- $\beta 1 /$ Smad3 signaling pathway.

Transformation of a non-motile polarized epithelial phenotype to a motile and invasive mesenchymal phenotype is frequently correlated with a high risk of metastasis and poor prognosis (20). The cadherin switch during EMT in NSCLC is regarded as a hallmark of invasiveness and metastasis. E-cadherin is a cell-cell adhesion molecule and EMT is characterized by E-cadherin loss. Reduced E-cadherin expression promotes cell mobility and invasion due to several transcriptional repressors, including ZEB1, Twist, Slug and Snail. EMT-related protein alteration is a significant prognostic marker to predict overall survival in patients with lung adenocarcinoma (21). Snail and Slug, which are zinc finger factors, are overexpressed in epithelial cell lines. Slug is a direct repressor of E-cadherin through E-box interaction and promotes cell invasion and metastasis. ZEB1 is enhanced by Slug (22). Our finding presents compelling evidence that nobiletin effectively reverses the acquisition of mesenchymal characteristics and suppresses the expression of transcription factors in TGF- $\beta$-stimulated lung cancer cells. The key mechanism that we elucidated to account for this important novel function of nobiletin is that it inhibits the transcriptional activity of Smad3.

Ligand binding to TGF- $\beta$ receptors initiates Smad2/3/4 complex formation. Smads are mainly localized in the cytoplasm. After the binding of TGF- $\beta$ to the receptor and subsequent TGF- $\beta R 1$ phosphorylation, trimers consisting of phosphorylated-Smad2/3 and Smad4 translocate to the nucleus, where they cooperate with transcription factors such as Snail and Twist to repress the expression of epithelial markers and activate the expression of mesenchymal markers at the mRNA level (23). This signaling is referred to as TGF- $\beta$-activated Smad signaling in EMT. Smad3 is regarded as a positive regulator of EMT while Smad2 is known as a negative mediator (24). In line with previous research, the primary role of Smad3 was confirmed in the present study. Mesenchymal markers such as N-cadherin, Snail and ZEB1 were downregulated due to Smad3 siRNA transfection of A549 cells. However, Smad2 siRNA had little or negligible effect on the expression of EMT-related proteins. Nobiletin influenced neither the expression of phospho-Smad nor Smad translocation into the nucleus. However, the transcriptional activity of Smad3 was almost abolished following nobiletin treatment. Moreover, the imbalanced promoter expression of E-cadherin and $\mathrm{N}$-cadherin were brought to a normal level by nobiletin treatment. Collectively, these data suggest that nobiletin targets Smad3 to suppress EMT.

MMP-2 and MMP-9 have been shown to promote cell invasion and metastasis of NSCLC. MMPs have also been identified as another class of inducers that activate developmental EMT. MMPs remodel the extracellular matrix to facilitate EMT and to promote cell specification during embryonic development. Furthermore, MMP-2 was reported to be involved in the suppressive effect of nobiletin on tumor formation and metastasis in a nasopharyngeal cancer xenograft model in vivo (25). Moreover, nobiletin inhibited tumor cell invasive activity not only by suppressing MMP-9 but also augmenting TIMP-1 production in human fibrosarcoma HT-1080 cells (26). EMT transcription factors stimulate MMP expression, leading to an increase in MMPs, which further activates EMT transcription factors in a feed-forward loop (27). TGF- $\beta$ signaling regulates the balance of MMP/TIMP to control matrix homeostasis (28). Recent evidence indicates that the recruitment of MMP-9 to the tumor cell surface triggers TGF- $\beta$ activation and enhances tumor growth and invasion (29). TGF- $\beta 1$ incubation resulted in an obvious surge in both the mRNA and protein levels of MMP-2 and MMP-9, while a significant reduction in MMP-2 and MMP-9 levels was observed due to nobiletin treatment.

High expression of FAK protein is reported in many cancers (30). A novel function for paxillin, Src and FAK has been discovered in mediating EMT and aggressiveness regulated by TGF- $\beta$ (30-32). FAK can be activated by a reduction in E-cadherin to facilitate focal adhesion assembly (33). FAK correlates with integrin clustering at the site of focal adhesions. As an oncogene involved in cell migration and in lung cancer tissues, paxillin is also highly expressed, amplified, and associated with EMT (34). In addition, Src-mediated regulation of E-cadherin and vimentin has been described in several cancers (35). Moreover, EMT is associated with acquired resistance to the EGFR inhibitor. Inhibition of the Src/FAK pathway 
contributed to overcome the EMT-associated resistance to erlotinib (36). In line with results revealing TGF- $\beta 1$-mediated upregulation of Src, FAK and paxillin, TGF- $\beta 1$ in A549 cells strongly induced the phosphorylation of Src, FAK and paxillin, and nobiletin markedly inhibited the induction.

In summary, abrogation of TGF- $\beta$-induced EMT may be an appropriate therapy to block metastasis. The present study provides compelling evidence that nobiletin exerts modulatory effects on TGF- $\beta$ signaling and its downstream responses, not only in vitro but also in metastatic experimental models in vivo. Nobiletin may be advanced as a new therapy to treat NSCLC metastasis and EMT-related disorders.

\section{References}

1. Barton MK: Patients of all ages with advanced non-small cell lung cancer are not receiving chemotherapy. CA Cancer J Clin 65: 337-338, 2015.

2. Thiery JP, Acloque H, Huang RY and Nieto MA: Epithelialmesenchymal transitions in development and disease. Cell 139: 871-890, 2009.

3. Hou M, Cheng Z, Shen H, He S, Li Y, Pan Y, Feng C, Chen X, Zhang Y, Lin M, et al: High expression of CTHRC1 promotes EMT of epithelial ovarian cancer (EOC) and is associated with poor prognosis. Oncotarget 6: 35813-35829, 2015.

4. Micalizzi DS, Farabaugh SM and Ford HL: Epithelialmesenchymal transition in cancer: Parallels between normal development and tumor progression. J Mammary Gland Biol Neoplasia 15: 117-134, 2010.

5. Ma L, Teruya-Feldstein J and Weinberg RA: Tumour invasion and metastasis initiated by microRNA-10b in breast cancer. Nature 449: 682-688, 2007.

6. Iwatsuki M, Mimori K, Yokobori T, Ishi H, Beppu T, Nakamori S, Baba $\mathrm{H}$ and Mori $\mathrm{M}$ : Epithelial-mesenchymal transition in cancer development and its clinical significance. Cancer Sci 101: 293-299, 2010

7. Kim YJ, Choi WI, Jeon BN, Choi KC, Kim K, Kim TJ, Ham J, Jang HJ, Kang KS and Ko H: Stereospecific effects of ginsenoside $20-\mathrm{Rg} 3$ inhibits TGF- $\beta 1$-induced epithelial-mesenchymal transition and suppresses lung cancer migration, invasion and anoikis resistance. Toxicology 322: 23-33, 2014.

8. Cichon MA and Radisky DC: ROS-induced epithelial-mesenchymal transition in mammary epithelial cells is mediated by $\mathrm{NF}-\kappa \mathrm{B}-$ dependent activation of Snail. Oncotarget 5: 2827-2838, 2014.

9. Kim J, Moon SH, Kim BT, Chae CH, Lee JY and Kim SH: A novel aminothiazole KY-05009 with potential to inhibit Traf2-and Nck-interacting kinase (TNIK) attenuates TGF- $\beta 1$ mediated epithelial-to-mesenchymal transition in human lung adenocarcinoma A549 cells. PLoS One 9: e110180, 2014.

10. Fong YC, Hsu SF, Wu CL, Li TM, Kao ST, Tsai FJ, Chen WC, Liu SC, Wu CM and Tang CH: Transforming growth factorbetal increases cell migration and betal integrin up-regulation in human lung cancer cells. Lung Cancer 64: 13-21, 2009.

11. Ko H: Geraniin inhibits TGF- $\beta 1$-induced epithelial-mesenchymal transition and suppresses A549 lung cancer migration, invasion and anoikis resistance. Bioorg Med Chem Lett 25: 3529-3534, 2015.

12. Nogata Y, Sakamoto K, Shiratsuchi H, Ishii T, Yano M and Ohta H: Flavonoid composition of fruit tissues of citrus species. Biosci Biotechnol Biochem 70: 178-192, 2006.

13. Lee YC, Cheng TH, Lee JS, Chen JH, Liao YC, Fong Y, Wu CH and Shih YW: Nobiletin, a citrus flavonoid, suppresses invasion and migration involving FAK/PI3K/Akt and small GTPase signals in human gastric adenocarcinoma AGS cells. Mol Cell Biochem 347: 103-115, 2011.

14. Baek SH, Kim SM, Nam D, Lee JH, Ahn KS, Choi SH, Kim SH, Shim BS, Chang IM and Ahn KS: Antimetastatic effect of nobiletin through the down-regulation of $\mathrm{CXC}$ chemokine receptor type 4 and matrix metallopeptidase-9. Pharm Biol 50: 1210-1218, 2012.

15. Gao XJ, Liu JW, Zhang QG, Zhang JJ, Xu HT and Liu HJ: Nobiletin inhibited hypoxia-induced epithelial-mesenchymal transition of lung cancer cells by inactivating of Notch-1 signaling and switching on miR-200b. Pharmazie 70: 256-262, 2015.
16. Song J, Shu L, Zhang Z, Tan X, Sun E, Jin X, Chen Y and Jia X: Reactive oxygen species-mediated mitochondrial pathway is involved in Baohuoside I-induced apoptosis in human non-small cell lung cancer. Chem Biol Interact 199: 9-17, 2012.

17. Yang M, Xie X, Ding Y: SALL4 is a marker of poor prognosis in serous ovarian carcinoma promoting invasion and metastasis. Oncol Rep 35: 1796-1806, 2016.

18. Smith AL, Robin TP and Ford HL: Molecular pathways: Targeting the TGF- $\beta$ pathway for cancer therapy. Clin Cancer Res 18: 4514-4521, 2012.

19. Moustakas A and Heldin $\mathrm{CH}$ : Signaling networks guiding epithelial-mesenchymal transitions during embryogenesis and cancer progression. Cancer Sci 98: 1512-1520, 2007.

20. Puisieux A, Brabletz T and Caramel J: Oncogenic roles of EMT-inducing transcription factors. Nat Cell Biol 16: 488-494, 2014.

21. Shi Y, Wu H, Zhang M, Ding L, Meng F and Fan X: Expression of the epithelial-mesenchymal transition-related proteins and their clinical significance in lung adenocarcinoma. Diagn Pathol 8: 89, 2013.

22. Bolós V, Peinado H, Pérez-Moreno MA, Fraga MF, Esteller M and Cano A: The transcription factor Slug represses E-cadherin expression and induces epithelial to mesenchymal transitions: A comparison with Snail and E47 repressors. J Cell Sci 116: 499-511, 2003.

23. Kim J and Hwan Kim S: CK2 inhibitor CX-4945 blocks TGF- $\beta 1$ induced epithelial-to-mesenchymal transition in A549 human lung adenocarcinoma cells. PLoS One 8: e74342, 2013.

24. Hoot KE, Lighthall J, Han G, Lu SL, Li A, Ju W, Kulesz-Martin M, Bottinger E and Wang XJ: Keratinocyte-specific Smad2 ablation results in increased epithelial-mesenchymal transition during skin cancer formation and progression. J Clin Invest 118: 2722-2732, 2008.

25. Chien SY, Hsieh MJ, Chen CJ, Yang SF and Chen MK: Nobiletin inhibits invasion and migration of human nasopharyngeal carcinoma cell lines by involving ERK1/2 and transcriptional inhibition of MMP-2. Expert Opin Ther Targets 19: 307-320, 2015.

26. Sato T, Koike L, Miyata Y, Hirata M, Mimaki Y, Sashida Y, Yano $\mathrm{M}$ and Ito $\mathrm{A}$ : Inhibition of activator protein-1 binding activity and phosphatidylinositol 3-kinase pathway by nobiletin, a polymethoxy flavonoid, results in augmentation of tissue inhibitor of metalloproteinases-1 production and suppression of production of matrix metalloproteinases- 1 and -9 in human fibrosarcoma HT-1080 cells. Cancer Res 62: 1025-1029, 2002.

27. De Craene B and Berx G: Regulatory networks defining EMT during cancer initiation and progression. Nat Rev Cancer 13: 97-110, 2013.

28. Su BH, Tseng YL, Shieh GS, Chen YC, Wu P, Shiau AL and Wu C: Overexpression of prothymosin $\alpha$ antagonizes TGF- $\beta$ signalling to promote the development of emphysema. J Pathol 238: 412-422, 2015.

29. Dayer $\mathrm{C}$ and Stamenkovic I: Recruitment of matrix metalloproteinase-9 (MMP-9) to the fibroblast cell surface by lysyl hydroxylase 3 (LH3) triggers transforming growth factor- $\beta$ (TGF- $\beta$ ) activation and fibroblast differentiation. J Biol Chem 290: 13763-13778, 2015.

30. Cicchini C, Laudadio I, Citarella F, Corazzari M, Steindler C, Conigliaro A, Fantoni A, Amicone L and Tripodi M: TGFbetainduced EMT requires focal adhesion kinase (FAK) signaling. Exp Cell Res 314: 143-152, 2008.

31. Baquero P, Jiménez-Mora E, Santos A, Lasa M and Chiloeches A: TGF $\beta$ induces epithelial-mesenchymal transition of thyroid cancer cells by both the BRAF/MEK/ERK and Src/FAK pathways. Mol Carcinog: Sep 21, 2015 (Epub ahead of print). doi: $10.1002 / \mathrm{mc} .22415$.

32. Guarino M: Src signaling in cancer invasion. J Cell Physiol 223: 14-26, 2010.

33. Frame MC and Inman GJ: NCAM is at the heart of reciprocal regulation of E-cadherin- and integrin-mediated adhesions via signaling modulation. Dev Cell 15: 494-496, 2008.

34. Jagadeeswaran R, Surawska H, Krishnaswamy S, Janamanchi V, Mackinnon AC, Seiwert TY, Loganathan S, Kanteti R, Reichman T, Nallasura V, et al: Paxillin is a target for somatic mutations in lung cancer: Implications for cell growth and invasion. Cancer Res 68: 132-142, 2008.

35. Nagathihalli NS and Merchant NB: Src-mediated regulation of E-cadherin and EMT in pancreatic cancer. Front Biosci 17: 2059-2069, 2012.

36. Wilson C, Nicholes K, Bustos D, Lin E, Song Q, Stephan JP, Kirkpatrick DS and Settleman J: Overcoming EMT-associated resistance to anti-cancer drugs via Src/FAK pathway inhibition. Oncotarget 5: 7328-7341, 2014. 